\title{
Cociente emocional de los estudiantes de Administración y Gestión Comercial de una universidad privada
}

\author{
Lita Regina Rojas Samanez ${ }^{1 a}$, Dennis Arias Chávez ${ }^{2}$, Roger Pedro Norabuena \\ Figueroa $^{3}$, Augusto César Mescua Figueroa ${ }^{4}$, Jessica Paola Palacios Garay y María \\ Jesús Caurcel Cara ${ }^{6}$
}

\author{
Universidad César Vallejo, Lima, Perú ${ }^{145}$ \\ Universidad Continental, Arequipa, Perú ${ }^{2}$ \\ Universidad Nacional Santiago Antúnez de Mayolo, Huaraz, Perú ${ }^{3}$ \\ Universidad de Granada, Granada, España ${ }^{6}$ \\ Orcid ID: https://orcid.org/0000-0001-8365-9465 \\ Orcid ID: https://orcid.org/0000-0003-1500-8366 \\ Orcid ID: https://orcid.org/0000-0003-3731-98433 \\ Orcid ID: https://orcid.org/0000-0002-6812-24994 \\ Orcid ID: https://orcid.org/0000-0002-2315-1683 \\ Orcid ID: https://orcid.org/0000-0002-2169-91006
}

Recibido: 23 de abril de 2018

Aceptado: 15 de diciembre 2018

\section{Resumen}

La investigación tiene como objetivo determinar la dimensión predominante del cociente emocional de los estudiantes de Administración y Gestión Comercial de una universidad peruana privada. La investigación es de tipo sustantiva y de diseño no experimental, de nivel descriptivo-explicativo y de corte transversal. El instrumento utilizado para evaluar la variable ha sido el cuestionario del cuadro de Cociente Emocional (CE) (Cooper y Sawaf, 1997), el cual está conformado por 262 preguntas aplicadas sobre una muestra de 86 estudiantes universitarios. Los resultados permitieron determinar que la dimensión "Valores y creencias" $(\mathrm{B}=1.814)$ presenta mayor coeficiente y, por ende, aporta más al cociente emocional de los estudiantes analizados. Esta predominancia parte del resultado de la dimensión que presenta un odds ratio $(\operatorname{Exp}(B))=6.134$, lo que evidencia que esta es una dimensión de riesgo, lo que llevaría a que un estudiante tenga 6.134 de veces de 
posibilidad de presentar niveles bajos de cociente emocional respecto a otro con niveles altos de cociente emocional por causa de la dimensión "Valores y creencias".

Palabras clave: Cociente emocional, inteligencia emocional, liderazgo, estudiantes universitarios.

\title{
Emotional quotient of the Administration and Commercial Management students of a private university
}

\begin{abstract}
This research study's objective is to determine the predominant dimension in the emotional quotient of the Business Management and Administration students of a private university of Peru. The research was substantive, non-experimental, descriptiveexplanatory and cross-sectional. The instrument used to evaluate the variable was the of Emotional Quotient questionnaire (Cooper \& Sawaf, 1997), which is composed of 262 questions, and it was applied on a sample of 86 university students. The results show that the values and beliefs dimension $(\mathrm{B}=1.814)$ has a higher coefficient and, thus, contributes more to the emotional quotient of the students analyzed. This dominant part of the results of the dimension presented an odds ratio $(\operatorname{Exp}(B))=6.134$, which shows that this is a dimension of risk, which would lead a student to have 6.134 times greater possibility of presenting low levels of emotional quotient with respect to another with high levels of emotional quotient because of the dimension values and beliefs.
\end{abstract}

Keywords: Emotional quotient, emotional intelligence, leadership, college and university students.

\section{Introducción}

Salovey y Mayer (1990) definen la inteligencia emocional como la habilidad para manejar los sentimientos y emociones, discriminar entre ellos y utilizar estos para dirigir los propios pensamientos y acciones. Este conocimiento permite desarrollar habilidades dentro de la vida diaria con el fin de mejorar y promover la inteligencia natural. Por su parte, Goleman (1996) considera que la inteligencia emocional es "aquella capacidad que tienen los seres humanos para tomar conciencia de sus emociones, comprender la de los demás, tolerar presiones y frustraciones laborales, habilidad para trabajar en equipo y adoptar una posición empática que ayude al desarrollo y éxito personal” (p.54).

Ahora bien, el conocimiento de la educación emocional da respuesta a varias necesidades sociales, las cuales no son atendidas dentro de la educación formal de las personas. Existen muchos problemas que afectan a la sociedad actual, por ejemplo, el consumo de drogas, alcohol, violencia, prejuicios étnicos, etcétera. Todo esto tiene un fondo emocional, por lo que, se necesitan hacer cambios en el sentido socio emocional 
con el fin de prevenir estos comportamientos de riesgo. La solución a esta problemática puede ser la educación emocional y sus cuotas de prevención hacia estas emociones débiles.

La competencia emocional es un constructo amplio que incluye diversos procesos que tienen una variedad de consecuencias. Adquirirla implica dominar un conjunto de conocimientos, capacidades, habilidades y actitudes muy necesarias para comprender y expresar las situaciones o fenómenos emocionales. Autores como Salovey y Slayter (1997) identifican cinco dimensiones básicas dentro de la competencia emocional: (a) Cooperación, (b) Asertividad, (c) Responsabilidad, (d) Empatía y (e) Autocontrol. Estas dimensiones son coherentes con el concepto de inteligencia emocional la cual incluyen, a su vez, dimensiones como (a) Autoconciencia emocional, (b) Manejo de las emociones, (c) Motivación, (d) Empatía y (d) Habilidades sociales (Blanco, 2007).

En la actualidad, las competencias emocionales son consideradas como un aspecto muy importante en la captación de personal para las empresas debido a que en el ambiente laboral y productivo se buscan cada vez más personas emocionalmente competentes (Cherniss, 2000; Elias et al., 1997; Goleman, Boyatzis y Mckee, 2002).

\section{Dimensiones de cociente emocional}

A continuación, se desarrolla un breve esbozo de cada una de las dimensiones del cociente emocional.

Dimensión Ambiente actual: esta dimensión hace alusión a las circunstancias de vida actual y el ambiente que rodean al individuo, ya sea en el hogar como en el lugar de trabajo. Abarca tres escalas: sucesos en la vida, presiones del trabajo y presiones personales. Los sucesos de vida se refieren al grado en que el individuo se ve afectado emocionalmente y experimentando las ocurrencias que pueden producirle estrés como, por ejemplo, un déficit económico y seguridad en cuanto a vivienda, la muerte de un ser querido o algún conflicto familiar.

Las presiones de trabajo medirán el grado en que se verán afectadas las emociones ante las circunstancias y acontecimientos que pueden suceder dentro la vida laboral del individuo, como por ejemplo la inseguridad de mantener el empleo, el tipo de relación con sus compañeros de trabajo, etapas de trabajo bajo presión, etc. En el indicador "Presiones personales" se evalúa el grado en que afectarán las emociones del individuo y 
las situaciones que genere alguna presión de índole personal producido por situaciones de estrés laboral familiar o netamente personal.

Dimensión Conocimientos: la dimensión conocimiento emocional se refiere a la conciencia o conocimiento básico que un individuo puede tener acerca de sus emociones y los de otras personas, esto significa entender grandemente cómo funcionan las emociones, las fortalezas, las debilidades, las necesidades e impulsos de los individuos, se puede decir que estas personas con buen nivel de autoconocimiento o autoconciencia no son demasiado exigentes y no tienen esperanzas irreales, por el contrario, presentan bajos niveles de opiniones críticas y depresión ante lo externo ya que reconocen como sus sentimientos y sus emociones pueden afectarles a ellos mismos, a las personas de su entorno y el desempeño que pueden tener en su centro de labores.

Esta dimensión trata sobre lo que contienen las emociones y habilidades para así poder direccionarlas y utilizarlas de forma adecuada y exitosa. Dentro de esta dimensión figuran los siguientes indicadores: conocimiento emocional de sí mismo, expresión emocional y conocimiento emocional de otros. En el conocimiento emocional de sí mismo se evalúa el grado en que el individuo posee conocimiento de su propia emocionalidad, es decir, conciencia de sus emociones y sentimientos. En expresión emocional se evalúa que tanto el individuo puede expresar de forma positiva o negativa sus emociones. En el indicador conciencia emocional de los otros se evalúa el nivel de conciencia emocional que se adquiere de las personas con quienes interactúa, es decir, la capacidad que posee el individuo para escuchar, sentir o intuir lo que las personas a su alrededor están sintiendo a través de diferentes formas de comunicación como, por ejemplo, palabras o lenguaje corporal.

Dimensión Competencias: esta dimensión busca determinar el potencial que tiene cada individuo para aprender y adquirir ciertas habilidades o competencias emocionales. Esto va a mostrar hasta qué punto un individuo puede trasladar su potencial a sus actividades diarias. Esta dimensión consta de cinco escalas que exploran las habilidades y patrones de conducta que se han desarrollado a lo largo del tiempo para enfrentar y responder ante las circunstancias de vida. Estas escalas son: la intención, la creatividad, la flexibilidad, las conexiones interpersonales y el descontento constructivo.

El indicador intención explora el deseo o propósito del acto deliberado de un individuo, esto para expresarse, decir lo que piensa y hacer lo que se dice; la escala mide 
el grado de conciencia en que se pueden tomar decisiones coherentes con los objetivos y valores de las personas. El indicador creatividad se refiere a la capacidad de concebir nuevas ideas ofrecer soluciones alternativas y opciones de realizar sus actividades. El indicador elasticidad se refiere a la capacidad de adaptarse a las condiciones y situaciones cambiantes de la vida diaria considerando las reacciones de frustración o decepción a nivel laboral, la paciencia e incluso del humor ante situaciones adversas. La dimensión conexiones interpersonales explora si un individuo es capaz de buscar y mantener relaciones interpersonales con las que se sienta cómodo y libre. En el indicador descontento constructivo se evalúa que tanto el sujeto puede permanecer tranquilo concentrado ante situaciones difíciles o conflictivas mostrando serenidad y autocontrol.

Dimensión Valores y Creencias: son los patrones y principios de pensamientos y sentimientos que dirigen las acciones del individuo. Consta de seis indicadores que desean explorar las profundas formas de sentir que influencian en las acciones en la vida personal y como en el ámbito laboral de las personas. El indicador compasión se refiere al alto nivel de empatía, aprecio y respeto por las posiciones o circunstancias en que se encuentran las otras personas, esto incluye la capacidad de perdonarse a uno mismo y a los demás. El indicador perspectiva estudia la forma de percepción del mundo alrededor y el lugar que el individuo ocupa dentro, se considera también la visión optimista o negativa ante los acontecimientos adversos. El indicador intuición se refiere a la visión o sensaciones que no sean respaldadas por investigaciones o hechos reales sino, más bien, se refiere a las reacciones intuitivas en sus posiciones y toma de decisiones. El indicador ratio de confianza medirá el grado en que el individuo puede esperar de las demás personas un comportamiento justo y equitativo. El indicador poder personal hace referencia a la capacidad de sentirse útil percibido y entusiasmado ante las circunstancias, también se evalúa el grado en que enfrenta los desafíos de la vida y por último el indicador integridad se referirá al grado en el que la persona encaja coherentemente sus acciones y sus formas de pensar y de sentir a nivel emocional intelectual y espiritual.

Dimensión Resultados: esta dimensión mide el impacto que ha tenido la inteligencia emocional en la vida del individuo de esta forma el test arroja un coeficiente que delibera el estado de salud física, emocional y psicológica, también puede verse la calidad de vida a través de síntomas. En el indicador calidad de vida se evalúa qué tanto desarrollo tiene el individuo con respecto de una alta calidad de vida emocional, satisfacción, pesimismo y los sentimientos involucrados en su ambiente laboral. El cociente de relaciones mide la 
capacidad de involucrarse con personas con quienes se comparte no solo el ambiente laboral sino también el social, y convertirse en una persona importante y útil. Por último, en el indicador óptimo rendimiento se evalúa la autosatisfacción de la persona con respecto a su desenvolvimiento en el área de trabajo, la forma en que percibe las comunicaciones, el estado de relaciones con sus compañeros y el grado de responsabilidad y compromiso.

\section{Metodología}

La presente investigación es de tipo sustantiva y de diseño no experimental, de nivel descriptivo-explicativo y de corte transversal. Se plantea el análisis de un conjunto de hipótesis fruto de la revisión teórica, basándose en el análisis de los resultados obtenidos de la medición entre las variables propuestas. Como se trata de una investigación con enfoque cuantitativo, se utiliza el cuestionario del cuadro de Cociente emocional (CE) elaborado por Cooper y Sawaf (1997), el cual está conformado por 262 preguntas. El instrumento fue estandarizado, no se hizo ninguna modificación y se aplicó en su totalidad tal como fue planteada por sus autores.

\section{Participantes}

En el estudio participan 86 estudiantes de la carrera de Administración y Gestión Comercial de una universidad privada peruana ubicada en Lima, elegidos por el método de muestreo de tipo no probabilístico denominado también como muestreo determinístico (Pérez-Tejada, 2008). La estrategia muestral seguida corresponde al tipo de muestreo por conveniencia, ya que se procedió a establecer contacto con la carrera profesional a través de su director quien autorizó la aplicación del instrumento. Con el fin de establecer mayor control se planteó como criterio de inclusión considerar como parte de la muestra solo a estudiantes regulares, esto por la naturaleza del instrumento que estudia al individuo en el contexto personal y ambiente de trabajo.

\section{Métodos de análisis de datos}

Realizada la recolección de datos, se puso en práctica el procesamiento, para lo cual se recurrió al uso del Software Estadístico SPSS en la versión 23.0, ejecutándose un análisis descriptivo, para el que se tomaron los datos distribuyéndose en tablas de frecuencias, que refieren a cuadros en los que se organizan los datos para que sean de 
utilidad en la toma de decisiones; así como un análisis inferencial, aplicándose el procesamiento estadístico de correlación cuantificada realizando el proceso correspondiente a la regresión logística.

\section{Aspectos éticos}

Conforme a los atributos que le atañen a la presente investigación, se tomaron en consideración los aspectos éticos fundamentales que responden a los hechos en su veracidad a la base de datos obtenida y los componentes teóricos que abordaron los autores citados siguiendo las normas APA en cada uno de los acápites que describe la variable "Cociente emocional".

\section{Resultados}

En la Tabla 1, se observa que existen diferencias en las dimensiones del cociente emocional de los estudiantes de Administración y Gestión Comercial de una universidad privada.

A la luz de los resultados, la dimensión "Valores y creencias" $(\mathrm{B}=1.814)$ presenta mayor coeficiente, por ende, aporta más al cociente emocional de los estudiantes de Administración y Gestión Comercial de la universidad estudiada. Así mismo, esta dimensión presenta un odds ratio $(\operatorname{Exp}(B))=6.134$, lo que significa que es una dimensión de riesgo, además de indicar que un estudiante tiene 6.134 de veces de posibilidad de presentar niveles bajos de cociente emocional respecto a otro estudiante con niveles altos de cociente emocional por causa de la dimensión valores y creencias.

Tabla 1

Coeficientes de la regresión logística de las dimensiones del cociente emocional de los estudiantes de Administración y Gestión Comercial de una universidad privada

\begin{tabular}{lcccccc}
\hline \multirow{2}{*}{ Dimensión } & \multirow{2}{*}{ B } & \multirow{2}{*}{ Error estándar } & \multirow{2}{*}{ Sig. } & \multirow{2}{*}{$\operatorname{Exp}(\mathbf{B})$} & \multicolumn{2}{c}{ 95\% C.I. para EXP(B) } \\
\cline { 5 - 7 } & & & & & Inferior & Superior \\
\hline Ambiente actual & 0.921 & 0.525 & 0.080 & 2.512 & 0.897 & 7.034 \\
Conocimiento & -1.508 & 0.942 & 0.109 & 0.221 & 0.035 & 1.402 \\
Competencias & 0.174 & 1.324 & 0.895 & 1.190 & 0.089 & 15.935 \\
Valores y creencias & 1.814 & 0.911 & 0.046 & 6.134 & 1.029 & 36.562 \\
Resultados & -0.415 & 0.838 & 0.621 & 0.660 & 0.128 & 3.415 \\
\hline Fuente propia
\end{tabular}

Fuente propia

El indicador de mayor peso son las "Presiones personales" (Tabla 2), pero esta, a su vez, no es estadísticamente significativa. No existen diferencias en los indicadores de la dimensión "Ambiente Actual" de los estudiantes analizados. 
El coeficiente de la regresión logística muestra el peso que tiene cada factor (dimensión o indicador) sobre la variable, ello significa que un coeficiente alto incrementa la probabilidad de que un encuestado (o evaluado) tenga un nivel bajo en la variable respecto a otro encuestado (o evaluado) que tenga un nivel alto en la variable.

Por otro lado, el valor de odds ratio $<\operatorname{Exp}(B)>$ muestra si el factor (o indicador) es protectora o de riesgo. Protectora si $\operatorname{Exp}(B)$ es menor a 1, riesgo si $\operatorname{Exp}(B)$ es mayor a 1, siempre y cuando cumpla en que la unidad no esté incluida en el intervalo de confianza del $\operatorname{Exp}(B)$.

Tabla 2

Coeficientes de la regresión logística de los indicadores de la dimensión "Ambiente Actual" los estudiantes de Administración y Gestión Comercial de una universidad privada es "Presiones personales"

\begin{tabular}{lcccccc}
\hline \multirow{2}{*}{ Indicador } & \multirow{2}{*}{ B } & \multirow{2}{*}{ Error estándar } & \multirow{2}{*}{ Sig. } & \multirow{2}{*}{$\operatorname{Exp}(\mathbf{B})$} & 95\% C.I. para EXP(B) \\
\cline { 5 - 7 } & & & & & Inferior & Superior \\
\hline Sucesos de la vida & -0.646 & 0.630 & 0.305 & 0.524 & 0.153 & 1.801 \\
Presiones del trabajo & -0.745 & 0.393 & 0.058 & 0.475 & 0.220 & 1.024 \\
Presiones personales & 0.784 & 0.552 & 0.155 & 2.190 & 0.743 & 6.462 \\
\hline Fuente propia & & & & & &
\end{tabular}

El indicador de mayor peso es la expresión emocional (Tabla 3), pero esta, a su vez, no es significativa estadísticamente. Por consiguiente, no existen diferencias en los indicadores de la dimensión "Conocimiento" en los estudiantes de Administración y Gestión Comercial de una universidad peruana privada. El valor de odds ratio $<\operatorname{Exp}(B)$ $>$ muestra si el factor (o indicador) es protectora o de riesgo. Protectora si Exp(B) es menor a 1, riesgo si $\operatorname{Exp}(\mathrm{B})$ es mayor a 1, siempre y cuando cumpla en que la unidad no esté incluida en el intervalo de confianza del $\operatorname{Exp}(B)$.

Tabla 3

Coeficientes de la regresión logística de los indicadores de la dimensión "Conocimiento" los estudiantes de Administración y Gestión Comercial de una universidad privada

\begin{tabular}{lcccccc}
\hline \multirow{2}{*}{ Indicador } & \multirow{2}{*}{ B } & $\begin{array}{c}\text { Error } \\
\text { estándar }\end{array}$ & Sig. & $\operatorname{Exp}(\mathbf{B})$ & \multicolumn{2}{c}{\begin{tabular}{c} 
95\% C.I. para \\
\cline { 5 - 7 }
\end{tabular}} \\
\cline { 5 - 7 } & & & & & Inferior & Superior \\
\hline Conciencia emocional de sí mismo & -0.054 & 0.871 & 0.951 & 0.948 & 0.172 & 5.220 \\
Expresión emocional & 1.694 & 1.016 & 0.095 & 5.441 & 0.743 & 39.828 \\
Conciencia emocional de otros & -0.493 & 0.844 & 0.559 & 0.611 & 0.117 & 3.192 \\
\hline
\end{tabular}

Fuente propia 
Como el valor de significación observada (Sig.) es menor al valor de significación teórica $\alpha=0.05$ en uno de los indicadores, se rechaza la hipótesis nula. Por consiguiente, existen diferencias en los indicadores de la dimensión "Competencias" los estudiantes de Administración y Gestión Comercial de una universidad privada (Tabla 4). Los resultados revelan que el indicador elasticidad $(B=4.271)$ presenta mayor coeficiente y por ende aporta más a la dimensión "Competencias" del cociente emocional de los estudiantes de Administración y Gestión Comercial de una universidad privada. Así mismo, este indicador presenta un odds ratio $(\operatorname{Exp}(B))=71.624$, significando que es un indicador de riesgo y además significa que un estudiante tiene 71.624 veces de posibilidad de presentar niveles bajos en la dimensión "Competencias" del cociente emocional respecto a otro estudiante con niveles altos en la dimensión "Competencias" del cociente emocional por causa del indicador elasticidad.

Tabla 4

Coeficientes de la regresión logística de los indicadores de la dimensión "Competencias" los estudiantes de Administración y Gestión Comercial de una universidad privada

\begin{tabular}{lcccccc}
\hline \multirow{2}{*}{ Dimensión } & \multirow{2}{*}{ B } & Error estándar & Sig. & $\operatorname{Exp}(\mathbf{B})$ & \multicolumn{2}{c}{$\mathbf{9 5 \%}$ C.I. para EXP(B) } \\
\hline Intención & -2.767 & 1.526 & 0.070 & 0.063 & 0.003 & 1.250 \\
Creatividad & 1.829 & 0.982 & 0.063 & 6.228 & 0.908 & 42.699 \\
Elasticidad & 4.271 & 1.922 & 0.026 & 71.624 & 1.657 & 3096.103 \\
Conexiones interpersonales & -0.593 & 1.292 & 0.646 & 0.553 & 0.044 & 6.955 \\
Descontento constructivo & -1.254 & 1.539 & 0.415 & 0.285 & 0.014 & 5.828 \\
\hline
\end{tabular}

Fuente propia

Como el valor de significación observada (Sig.) es menor al valor de significación teórica $\alpha=0.05$ en uno de los indicadores, se rechaza la hipótesis nula. Por consiguiente existen diferencias en los indicadores de la dimensión "Valores y creencias" los estudiantes de Administración y Gestión Comercial de una universidad privada (Tabla 5). Como puede observarse, el indicador perspectiva $(B=4.181)$ presenta mayor coeficiente y por ende aporta más a la dimensión "valores y creencias" del cociente emocional de los estudiantes de Administración y Gestión Comercial de una universidad privada. Así mismo, este indicador presenta un odds ratio $(\operatorname{Exp}(B))=65.401$, significando que es un indicador de riesgo, y además, significa que un estudiante tiene 65.401 veces de posibilidad de presentar niveles bajos en la dimensión "Valores y creencias" del cociente emocional respecto a otro estudiante con niveles altos en la dimensión "Valores y creencias" del cociente emocional por causa del indicador perspectiva. 
Tabla 5

Coeficientes de la regresión logística de los indicadores de la dimensión "Valores y creencias" los estudiantes de Administración y Gestión Comercial de una universidad privada

\begin{tabular}{|c|c|c|c|c|c|c|}
\hline \multirow{2}{*}{ Indicador } & \multirow{2}{*}{$\mathbf{B}$} & \multirow{2}{*}{ Error estándar } & \multirow{2}{*}{ Sig. } & \multirow{2}{*}{$\operatorname{Exp}(B)$} & \multicolumn{2}{|c|}{ 95\% C.I. para $\operatorname{EXP(B)}$} \\
\hline & & & & & Inferior & Superior \\
\hline Compasión & -2.508 & 1.399 & .073 & .081 & .005 & 1.263 \\
\hline Perspectiva & 4.18053 & 1.339 & .002 & 65.401 & 4.743 & 901.774 \\
\hline Intuición & .764 & 1.378 & .579 & 2.148 & .144 & 31.995 \\
\hline Radio de confianza & -.095 & 1.360 & .944 & 909 & .063 & 13.072 \\
\hline Poder personal & -.237 & 1.812 & .896 & .789 & .023 & 27.495 \\
\hline Integridad & -1.344 & 1.450 & .354 & .261 & .015 & 4.469 \\
\hline
\end{tabular}

Fuente propia

Como el valor de significación observada (Sig.) es menor al valor de significación teórica $\alpha=0.05$ en uno de los indicadores, se rechaza la hipótesis nula. Por consiguiente, existen diferencias en los indicadores de la dimensión "Resultados" los estudiantes de Administración y Gestión Comercial de una universidad privada (Tabla 6). Los resultados muestran que el indicador salud general $(B=1.214)$ presenta mayor coeficiente, y por ende aporta más a la dimensión "resultados" del cociente emocional de los estudiantes de Administración y Gestión Comercial de una universidad privada. Así mismo, este indicador presenta un odds ratio $(\operatorname{Exp}(B))=3.366$, significando que es un indicador de riesgo y además significa que un estudiante tiene 3.366 veces de posibilidad de presentar niveles bajos en la dimensión "Resultados" del cociente emocional respecto a otro estudiante con niveles altos en la dimensión "resultados" del cociente emocional por causa del indicador salud general.

Tabla 6

Coeficientes de la regresión logística de los indicadores de la dimensión "Resultados" los estudiantes de Administración y Gestión Comercial de una universidad privada

\begin{tabular}{lcccccc}
\hline \multirow{2}{*}{ Indicador } & \multirow{2}{*}{$\mathbf{B}$} & $\begin{array}{c}\text { Error } \\
\text { estándar }\end{array}$ & \multirow{2}{*}{ Sig. } & \multirow{2}{*}{$\operatorname{Exp}(\mathbf{B})$} & \multicolumn{2}{c}{$\mathbf{9 5 \%}$ C.I. para EXP(B) } \\
\cline { 6 - 7 } & & & & & Inferior & Superior \\
\hline Salud general & 1.214 & 0.490 & 0.013 & 3.366 & 1.289 & 8.793 \\
Calidad de vida & -0.167 & 0.525 & 0.750 & 0.846 & 0.302 & 2.367 \\
Cociente de relaciones & -0.738 & 0.464 & 0.112 & 0.478 & 0.193 & 1.187 \\
Óptimo rendimiento & 0.011 & 0.526 & 0.983 & 1.011 & 0.361 & 2.834 \\
\hline
\end{tabular}

Fuente propia

\section{Discusión de resultados}


Considerando el Cociente emocional como la capacidad de sentir, entender y aplicar eficazmente el poder y la agudeza de las emociones como fuente de energía humana, información, conexión e influencia, puedes decirse que los estudiantes se encuentran en un estado de transición y formación de inteligencia emocional aplicada a las organizaciones, lo que contrasta con la investigación de Rodríguez (2016) quien encontró 58 alumnos con un nivel alto de inteligencia emocional, 70 alumnos con un nivel medio y solo 2 alumnos que tienen un nivel bajo. Así mismo, existen 34 alumnos con un nivel alto de liderazgo, 92 alumnos que contaban un nivel medio y 4 alumnos que tienen un nivel bajo de liderazgo. Los alumnos son capaces de entablar buenas relaciones con los demás y de incidir en sus decisiones apoyándoles y orientándoles.

Similares resultados obtuvieron Peñalva, López y Landa (2013) quienes encontraron que uno de cada dos alumnos presenta un déficit en competencias emocionales que incluyen habilidades personales e interpersonales y que les lleva a tener miedo a hablar en público (43.6\%), a no sentirse capaz de liderar un grupo (40\%) o a no intentar asumir el mando e imponer un ritmo de trabajo cuando trabaja en equipo (60.9\%). Un punto importante es que se encontró que los alumnos no controlan en su totalidad sus emociones e impulsos, así mismo, no se encuentran totalmente motivados por lo que la intervención docente es fundamental, así como lo menciona Gaeta y López (2013) donde se evidencia la importancia de que el sistema universitario se adapte a los nuevos tiempos, lo que implica la necesidad de ampliar la información científica y técnica de los estudiantes en torno a las competencias emocionales a fin de preparar a los estudiantes para la vida, siendo de suma utilidad que los docentes diagnostiquen las competencias emocionales de los estudiantes, de manera que puedan delinear las actividades de forma más apropiada para obtener los máximos aprovechamientos.

En los resultados de la presente investigación se encontró que el 74\% de los estudiantes de Administración y Gestión Comercial de una universidad peruana privada presentan un nivel bajo de cociente emocional en la dimensión resultados, mientras que el $1 \%$ de los mismos presenta un nivel alto. Esta dimensión mide el impacto que la inteligencia emocional está teniendo actualmente en la vida de la persona. El coeficiente emocional se hace palpable en nuestra salud física, emocional y psicológica y la calidad de vida es afectada. Sus indicadores son la salud general, la calidad de vida, el cociente de relaciones y el óptimo rendimiento. Similares resultados podemos ver en la investigación de Peñalva, López y Landa (2012) en la que se concluyó que uno de cada dos alumnos presenta un déficit en competencias emocionales que incluyen habilidades 
personales e interpersonales y que les lleva a tener miedo a hablar en público (43.6\%), a no sentirse capaz de liderar un grupo (40\%) o a no intentar asumir el mando e imponer un ritmo de trabajo cuando trabaja en equipo (60.9\%), por lo que no se están teniendo los resultados óptimos de un cociente emocional favorable. En contraposición se encuentra el caso de la investigación de Bustamante, Jiménez y Ruiz (2012) donde el clima organizacional ayudaba al bienestar emocional y a la percepción favorable que tienen los docentes sobre su lugar de trabajo, conllevando así a optimizar el desempeño general de todos ellos encaminados a generar mejor calidad en la educación y mostrar resultados positivos que fortalecen la imagen corporativa de la universidad como ente educativo de calidad.

La hipótesis general señala que la dimensión predominante del cociente emocional de los estudiantes de Administración y Gestión Comercial de una universidad privada es "Competencias". La dimensión competencias determina el potencial que tiene cada individuo para aprender y adquirir ciertas habilidades o competencias emocionales. Según los resultados de la investigación, la dimensión "Valores y creencias" ( $\mathrm{B}=1.814)$ presenta mayor coeficiente y por ende aporta más al cociente emocional de los estudiantes. Así mismo, esta dimensión presenta un odds ratio $(\operatorname{Exp}(B))=6.134$, significando que es una dimensión de riesgo, además de indicar que un estudiante tiene 6.134 de veces de posibilidad de presentar niveles bajos de cociente emocional respecto a otro estudiante con niveles altos de cociente emocional por causa de la dimensión valores y creencias. Por lo tanto, se rechaza la hipótesis general de investigación, ya que la dimensión predominante son los valores y creencias.

La dimensión "Valores y creencias" son patrones y principios de pensamientos y sentimientos que dirigen las acciones del individuo. Estos hallazgos coinciden con lo encontrado en el estudio de Bustamante, Jiménez y Ruiz (2012) en la que, producto de un buen clima organizacional, los docentes de la universidad del Quindío demuestran integridad en su actividad laboral, la cual es extensiva a su sistema de valores lo cual se ve reflejado en la confianza que brindan a sus estudiantes. Estos aspectos contribuyen en su labor educativa ya que muestra resultados positivos que fortalecen la imagen corporativa de la universidad como ente educativo de calidad.

La hipótesis específica 1 resalta que el indicador predominante dentro de la dimensión "Ambiente Actual" en los estudiantes analizados es "Presiones personales". Este indicador hace referencia al grado en que afectarán las emociones del individuo y las situaciones que genere alguna presión de índole personal producido por situaciones 
de estrés laboral familiar o netamente personal. Los resultados de la investigación indican que no existen diferencias en los indicadores de la dimensión "Ambiente actual". Por lo tanto, se rechaza la primera hipótesis específica de investigación, ya que el indicador de mayor peso son las presiones personales, aunque esta, a su vez, no es estadísticamente significativa.

Se puede inferir entonces que todos los indicadores de esta dimensión son igual de importantes para el equilibrio emocional de los estudiantes en función a su ambiente y contexto actual. Estos resultados se aproximan a las conclusiones llegadas por FragosoLuzuriaga (2015) quien concluye que para analizar las competencias emocionales deben de existir parámetros de dominio en relación con el contexto del sujeto. Este mismo autor recoge los comentarios de Abraham (2004) y Oberst, Josep y Vilaregut (2009), autores que consideran que la inteligencia emocional es "potencialidad" y que necesita de la estimulación del ambiente para manifestarse. Del mismo modo, Bustamante, Jiménez y Ruiz (2012) hacen alusión al buen entorno laboral como factor favorable en el desempeño de los docentes de planta de la Universidad del Quindío generando mejor calidad en la educación y mostrando resultados positivos que fortalecen la imagen corporativa de la universidad como ente educativo de calidad. y se ve reflejado en la confianza que brindan a sus estudiantes, lo que contribuye a su labor educativa.

La hipótesis específica 2 resalta que el indicador predominante dentro de la dimensión "Conocimiento" los estudiantes de Administración y Gestión Comercial de una universidad privada es "Conciencia emocional de sí mismo". En el conocimiento emocional de sí mismos evalúa el grado en que el individuo posee conocimiento de su propia emocionalidad, es decir, conciencia de sus emociones. Según los resultados de la investigación no existen diferencias en los indicadores de la dimensión "Conocimiento" los estudiantes de Administración y Gestión Comercial de una universidad privada. Entonces se puede considerar que los tres indicadores, conocimiento emocional de sí mismo, expresión emocional y conocimiento emocional de otros, son igual de importantes en esta dimensión para lograr un buen nivel de cociente emocional.

Estos hallazgos coinciden con lo encontrado en el estudio de Gaeta y López (2013) donde se evidencia la importancia de que el sistema universitario amplíe la información científica y técnica de los estudiantes en torno a las competencias emocionales a fin de preparar a los estudiantes para la vida. Lo cual implica, a su vez, la búsqueda del desarrollo humano, desde un enfoque preventivo. Es de suma utilidad que los docentes diagnostiquen las competencias emocionales de los estudiantes, de manera que puedan 
delinear las actividades de forma más apropiada para obtener los máximos aprovechamientos. Asimismo, Martínez y Díaz (2014) encontraron que el alumnado es consciente de la importancia de la adquisición de competencias emocionales entre las que destacan el liderazgo o la cooperación para el mercado laboral por lo que consideran muy apropiado trabajar con este tipo de competencias en la Universidad y coincidiendo con Gaeta y López (2013) en la importancia de la intervención del profesorado en el proceso de adquisición de las competencias emocionales y la formación de las mismas. En una realidad más desarrollada de intervención de los docentes, Rodríguez (2016) encontró que los alumnos del séptimo y noveno ciclo semestre 2015-I de la escuela de administración de la universidad nacional de Trujillo, fueron capaces de conocer sus propios estados internos, de identificar sus emociones y consecuencias, y son conscientes de sus fortalezas y debilidades para entablar buenas relaciones con los demás y de incidir en sus decisiones apoyándoles y orientándoles, así también son capaces de comprender a los demás, reconocer sus sentimientos coincidiendo con el indicador conciencia emocional de otros.

La hipótesis específica 3 resalta que el indicador predominante dentro de la dimensión "Competencias" en los estudiantes analizados es "Intención”. Este indicador explora el deseo o propósito del acto deliberado de un individuo, esto para expresarse, decir lo que piensa y hacer lo que se dice; la escala mide el grado de conciencia en que se pueden tomar decisiones coherentes con los objetivos y valores de las personas. Según los resultados de la investigación existen diferencias en los indicadores de la dimensión “Competencias" en los estudiantes analizados. Según los resultados, el indicador "Elasticidad" ( $\mathrm{B}=4.271)$ presenta mayor coeficiente y, por ende, aporta más a la dimensión "Competencias" del cociente emocional de los estudiantes. Así mismo, este indicador presenta un odds ratio $(\operatorname{Exp}(B))=71.624$, lo que significa que es un indicador de riesgo y además evidencia que un estudiante tiene 71.624 veces de posibilidad de presentar niveles bajos en la dimensión "Competencias" del cociente emocional respecto a otro estudiante con niveles altos en la dimensión "Competencias" del cociente emocional por causa del indicador elasticidad.

Por lo tanto, se rechaza la tercera hipótesis específica de investigación, ya que el indicador predominante es la "Elasticidad". Este indicador se refiere a la capacidad de adaptarse a las condiciones y situaciones cambiantes de la vida diaria considerando las reacciones de frustración o decepción a nivel laboral, la paciencia e incluso del humor ante situaciones adversas. Se puede contrastar estos resultados con lo hallado por 
Rodríguez (2016) quien encontró que los alumnos eran capaces de entablar buenas relaciones con los demás, comprender y reconocer sus sentimientos además de saber cómo decir las cosas y cómo actuar al percibir y entender los distintos puntos de vista que estos, es decir adaptarse a las circunstancias. La necesidad e importancia de la elasticidad como indicador predominante nace también de la realidad en que no todos presentan niveles iguales de cociente emocional, tal como lo señalan Peñalva, López y Landa (2013) quienes concluyen que las competencias emocionales (sean estas personales o sociales) y las competencias de liderazgo son diferentes, y no se presentan igual en cada individuo. Este hecho genera diferentes perfiles de alumnos con unas habilidades y unas carencias concretas en las que habría que intervenir específicamente.

Se puede, por lo tanto, contrastar estas conclusiones con una realidad no tan lejana a los estudiantes, en referencia a la misma casa de estudios, tal como Gaeta y López (2013) resaltan la importancia de que el sistema universitario se adapte a los nuevos tiempos, lo que implica la necesidad de ampliar la información científica y técnica de los estudiantes en torno a las competencias emocionales a fin de preparar a los estudiantes para la vida.

La hipótesis específica 4 resalta que el indicador predominante dentro de la dimensión "Valores y creencias" en los estudiantes analizados en el presente estudio es "Poder personal". Este indicador hace referencia a la capacidad de sentirse útil, percibido y entusiasmado ante las circunstancias, también se evalúa el grado en que enfrenta los desafíos de la vida. En tanto que el indicador "Integridad" se refiriere al grado en el que la persona encaja coherentemente sus acciones y sus formas de pensar y de sentir a nivel emocional, intelectual y espiritual. Según los resultados de la investigación existen diferencias en los indicadores de la dimensión "Valores y creencias" en los estudiantes analizados. El indicador "Perspectiva" $(\mathrm{B}=4.181)$ presenta mayor coeficiente y por ende aporta más a la dimensión "Valores y creencias" del cociente emocional de los estudiantes.

Así mismo, este indicador presenta un odds ratio $(\operatorname{Exp}(B))=65.401$, significando que es un indicador de riesgo, lo que además lleva a considerar que un estudiante tiene 65.401 veces de posibilidad de presentar niveles bajos en la dimensión "Valores y creencias" del cociente emocional respecto a otro estudiante con niveles altos en la dimensión "Valores y creencias" del cociente emocional por causa del indicador "Perspectiva". Por lo tanto, se rechaza la cuarta hipótesis específica de investigación, ya que el indicador predominante es la "Perspectiva". 
Este indicador estudia la forma de percepción del mundo alrededor y el lugar que el individuo ocupa dentro de él. Se considera también la visión optimista o negativa ante los acontecimientos adversos. Estos hallazgos coinciden con lo encontrado en el estudio de Bustamante, Jiménez y Ruiz (2012) para quienes la estabilidad laboral, reconocimiento al desempeño docente y garantía de condiciones mínimas en lo referente a su trabajo generan una óptima perspectiva hacia el desempeño en la institución con excelentes resultados para las partes involucradas. Esta percepción favorable que tienen los docentes, conlleva a optimizar el desempeño general de ellos encaminados a generar mejor calidad en la educación y mostrar resultados positivos que fortalecen la imagen corporativa de la universidad como ente educativo de calidad.

La hipótesis específica 5 resalta que el indicador predominante dentro de la dimensión "Resultados" en los estudiantes analizados es "Calidad de vida". En este indicador se evalúa qué tanto desarrollo tiene el individuo al respecto de una alta calidad de vida emocional, satisfacción, pesimismo y los sentimientos involucrados en su ambiente laboral. Según los resultados de la investigación existen diferencias en los indicadores de la dimensión "Resultados" en los estudiantes. A la luz de los resultados, el indicador "Salud general" $(B=1.214)$ presenta mayor coeficiente $y$, por ende, aporta más a la dimensión "Resultados" del cociente emocional de los estudiantes de Administración y Gestión Comercial de una universidad privada. Así mismo, este indicador presenta un odds ratio $(\operatorname{Exp}(B))=3.366$, significando que es un indicador de riesgo y además significa que un estudiante tiene 3.366 veces de posibilidad de presentar niveles bajos en la dimensión "resultados" del cociente emocional respecto a otro estudiante con niveles altos en la dimensión "Resultados" del cociente emocional por causa del indicador salud general.

Por lo tanto, se rechaza la quinta hipótesis específica de investigación, ya que el indicador predominante es la salud general. Este indicador mide el impacto que ha tenido la inteligencia emocional en la vida del individuo. De esta forma el test arroja un coeficiente que delibera el estado de salud física, emocional y psicológica, también se verá la calidad de vida a través de síntomas. Complementando esta formación podemos mencionar a Bustamante, Jiménez y Ruiz (2012) quienes concluyeron que los docentes de planta de la Universidad del Quindío demuestran integridad en su trabajo, la cual es extensiva a su sistema de valores y se ve reflejado en la confianza que brindan a sus estudiantes, lo que, a la larga, contribuye a su labor educativa.

Ahora bien, Gaeta y López (2013) en sus estudios destacan la importancia de 
generar programas que busquen el desarrollo de las competencias emocionales en todas sus dimensiones particularmente para los estudiantes que inician una carrera universitaria, con la finalidad de orientar y facilitar la toma de decisiones y desempeño académico, así como contribuir a su mayor bienestar personal y social. En contraste a esto tenemos los resultados de Peñalva, López y Landa (2012) quienes evidencian que las competencias emocionales no se presentan igual en cada individuo. Esto genera diferentes perfiles de alumnos con unas habilidades y unas carencias concretas.

En líneas generales, se puede concluir que existen diferencias en las dimensiones del cociente emocional de los estudiantes de Administración y Gestión Comercial de una universidad peruana privada. La dimensión "Valores y creencias" $(\mathrm{B}=1.814)$ presenta mayor coeficiente y por ende aporta más al cociente emocional. Así mismo, esta dimensión presenta un odds ratio $(\operatorname{Exp}(B))=6.134$, lo que indica que es una dimensión de riesgo. Por lo tanto, se rechaza la hipótesis general de investigación, ya que la dimensión predominante son los valores y creencias. En vista de estos resultados es conveniente proponer algunas recomendaciones que permitan mejorar la calidad emocional de los estudiantes, como mayor capacitación e involucramiento de los docentes, estrategia de empoderamiento aplicado a los estudiantes con el fin de ayudarlo a que tomen mejores decisiones personales, realizar evaluaciones diagnosticas constantes de la población estudiantil en materia de salud emocional e implementar programas de acompañamiento docente-estudiantes.

\section{Referencias}

Abraham, R. (2004). Emotional competence as antecedent to performance: contingency dramework. Genetic, Social and General Pychology Monographs, 130(2), 117143 , Recuperado de http://www.bibliocatalogo.buap.mx:2260/ehost/pdfviewer/pdfviewer?vid=5\&sid $=8126 \mathrm{c} 503-0 \mathrm{~d} 02-47 \mathrm{db}-832 \mathrm{~b}-511 \mathrm{a} 2 \mathrm{ded} 371 \mathrm{~b} \% 40$ sessionmgr $112 \& \mathrm{hid}=101$

Blanco, A. (2007). Trabajadores Competentes. Introducción y reflexiones sobre la gestión de recursos humanos por competencias. Madrid: Esic Editorial.

Bustamante, C., Jiménez, A. y Ruiz, R. (2014). Coeficiente emocional de los docentes de planta de la universidad del Quindío. Recuperado de https://studylib.es/doc/144359/coeficiente-emocional-de-los-docentes-de-plantade

Cooper, R. y Sawaf, A. (1997). Estrategia emocional para ejecutivos. Barcelona: Martínez Roca. 
Cherniss, C. (2000). Social and Emotional Competence in the Workplace. En R. BarOn y J. D. A. Parker, The Handbook of Emotional Intelligence. Theory, Development, Assessment, and Application at Home, School, and in the Workplace. (pp. 433459). San Francisco, Ca: Jossey-Bass.

Elias, M. et al. (1997). Promoting social and emotional learning. Guidelines for Educators. Alexandria, Virginia: ASCD.

Fragoso-Luzuriaga, R. (2015). Inteligencia emocional y competencias emocionales en educación superior, ¿un mismo concepto? Revista Iberoamericana de Educación Superior, 6(16), 110-125.

Gaeta, M. y López, C. (abril-junio, 2013). Competencias emocionales y rendimiento académico en estudiantes universitarios. Revista Electrónica Interuniversitaria de Formación del Profesorado, 16(2), 13-25. Recuperado de http://www.redalyc.org/pdf/2170/217029557002.pdf

Goleman, D. (1996). Inteligencia Emocional. Buenos Aires: Vergara.

Goleman, D., Boyatzis, R. y McKee, A. (2002). El líder resonante crea más. Barcelona: Plaza y Jánes.

Martínez, M. y Díaz, M. (2014). Trabajo cooperativo y competencias emocionales: un tándem esencial en el aula universitaria. Recuperado de https://web.ua.es/en/ice/jornadas-redes-2014/documentos/comunicacionesposters/theme-3/388718.pdf

Oberst, U., Josep, N y Vilaregut, A. (2009). Training emotional and social in higher education: the seminar methodology. Higher Education in Europe, 34(3), 523533.

Peñalva, A., López, J. y Landa, N. (steiembre-diciembre, 2013). Competencias emocionales del alumnado de Magisterio: posibles implicaciones profesionales. Revista de Educación 362. Recuperado de http://www.mecd.gob.es/dctm/revistade-educacion/doi/362246.pdf?documentId=0901e72b8163e9b3

Pérez-Tejada, H. (2008). Estadística para las ciencias sociales, del comportamiento y de la salud (3. ${ }^{\mathrm{a}}$ ed.). México D. F.: Cengage Learning.

Rodríguez, E. M. R. (2016). Comprensión teórica y proceso metodológico de la investigación cualitativa. In Crescendo, 6(2), 169-183.

Salovey, P. y Mayer, J. (1990). Inteligencia emocional. Imaginación, Conocimiento y Personalidad, 9(3), 185-211. 